\title{
The Instability of Mucoid Pseudomonas aeruginosa: Fluctuation Test and Improved Stability of the Mucoid Form in Shaken Culture
}

\author{
By J. R. W. GOVAN, JANET A. M. FYFE AND CATHERINE McMILLAN \\ Department of Bacteriology, University of Edinburgh, Medical School, \\ Teviot Place, Edinburgh EH8 $9 A G$
}

(Received 13 July 1978)

\section{INTRODUCTION}

The first description of mucoid Pseudomonas aeruginosa by Sonnenschein (1927) also reported the segregation of non-mucoid revertants. Subsequently, the instability of mucoid strains isolated either from patients or in vitro, and cultured on agar or in broth, has been well documented (Henriksen, 1948; Zierdt \& Schmidt, 1964; Martin, 1973; Govan, 1975); little is known, however, of the mechanism underlying instability.

The ability to maintain $P$. aeruginosa in the mucoid state in vitro is essential in studies of this unusual form. Govan (1975) described improved stability when mucoid $P$. aeruginosa was grown in the presence of surfactants, such as lecithin or sodium deoxycholate. In this report we describe improved stability in nutrient broth cultures, suitably agitated, and suggest an explanation for the instability of the mucoid form. We report the use of a fluctuation test and growth rate experiments to indicate that, in unshaken broth cultures, instability can be explained on the basis of spontaneous mutation to the non-mucoid form, followed by a growth rate advantage for non-mucoid revertants. Under conditions where stability is improved, as in the case of shaken cultures, this growth rate advantage is not so apparent.

\section{METHODS}

Bacteria. Strain PAo568 is a mucoid variant of the non-mucoid Pseudomonas aeruginosa strain PAO381 (leu-38 str-6 FP2; Stanisich \& Holloway, 1969) and produces an acetylated alginate-like exopolysaccharide composed of mannuronic and guluronic acids (Govan \& Fyfe, 1978). A non-mucoid revertant, PAO547, was obtained by subculture from the spreading, non-mucoid growth which appeared at the edge of a mucoid colony after several days growth on nutrient agar at $37^{\circ} \mathrm{C}$. Strain PAO548 is a prototrophic derivative of PA0547 obtained by transduction using phage F116L (Krishnapillai, 1971) propagated on the strain PAO1 (Holloway, 1955).

Media. Nutrient broth (NB) was Oxoid no. 2 (code CM67) with $0.5 \%$ (w/v) yeast extract (Oxoid) and nutrient agar (NA) was Columbia Agar Base (Oxoid, CM331). Desoxycholate citrate agar (DCA) was Oxoid CM227. Minimal agar (MA) was that described by Vogel \& Bonner (1956).

Transduction. The method used was that described by Holloway et al. (1962).

Fluctuation test. Fluctuation analysis was based on the method described by Luria \& Delbruck (1943). A mucoid colony from an $18 \mathrm{~h}$ culture of PAO568, grown on DCA, was suspended in $2 \mathrm{ml}$ physiological saline and diluted in saline such that addition of $0.1 \mathrm{ml}$ of the suspension to $10 \mathrm{ml} \mathrm{NB}$ (contained in a $150 \mathrm{ml}$ bottle) produced an initial density of less than 10 cells $\mathrm{ml}^{-1}$. Cultures were incubated for $30 \mathrm{~h}$ at $37^{\circ} \mathrm{C}$ without shaking and then vortexed briefly to obtain a uniform suspension. The numbers of mucoid and nonmucoid colony-forming units were measured as follows. Samples $(0.2 \mathrm{ml})$ were diluted in physiological saline and $0.1 \mathrm{ml}$ was spread on NA and incubated for $18 \mathrm{~h}$ at $37^{\circ} \mathrm{C}$. The percentage of non-mucoid revertants was calculated from the average count obtained from three plates, at a dilution yielding 100 to 200 colonies per plate.

To investigate the stability of the mucoid form in shaken broth cultures, the inoculum was prepared as above but cultures were incubated in an orbital incubator (Gallenkamb) at 140 rev. min $^{-1}$. 
Growth rate experiment. The culture vessels and medium used were as described for the fluctuation test. Incubation was at $37^{\circ} \mathrm{C}$ and the culture vessels were shaken at $140 \mathrm{rev}$. $\mathrm{min}^{-1}$ or unshaken. Strains PAO568 and PAO548 were grown from single colonies, inoculated into NB and shaken for $18 \mathrm{~h}$ at $37^{\circ} \mathrm{C}$, diluted in physiological saline and inoculated together into $10 \mathrm{ml} \mathrm{NB}$ to give initial densities of $10^{6}$ and $10^{3} \mathrm{cells}^{-1}$, respectively. Viable counts of mucoid and non-mucoid colony-forming units were determined at $1.5 \mathrm{~h}$ intervals by removing $0.1 \mathrm{ml}$ from the culture, diluting appropriately in saline and plating $0.1 \mathrm{ml}$ in triplicate on NA and MA.

\section{RESUI.TS AND DISCUSSION}

Results of a fluctuation test showed that the mean percentage of non-mucoid revertants in 12 independent cultures of PAO568 was 37.8 with a variance of $390 \cdot 1$, whereas for 12 samples taken from a single culture, the mean percentage was 43.9 with a variance of 14.7 . This experiment clearly demonstrated the instability of PA0568 in unshaken NB cultures incubated for $30 \mathrm{~h}$ at $37^{\circ} \mathrm{C}$. In addition, the difference in variance between the 12 independent cultures and 12 samples from the same culture indicated a clonal distribution of non-mucoid revertants among the mucoid populations. In contrast, the percentage of non-mucoid revertants observed in each of 12 cultures of PAO568 inoculated in a similar manner but incubated with shaking for $30 \mathrm{~h}$ was less than $0.2 \%$.

An explanation for the instability of PAO568 could be that spontaneous mutations result in non-mucoid revertants and that revertants have a growth rate advantage over the mucoid parent strain which is more pronounced in unshaken than in shaken cultures. To test this hypothesis the growth rates of PAO568 and of a non-mucoid revertant were studied in a competitive situation (see Methods). The non-mucoid revertant used was the prototrophic transductant PAO548. This strain was derived for the experiment from the auxotrophic non-mucoid revertant PAO547 so that viable counts of the non-mucoid strain could be determined using unsupplemented MA plates and thus be distinguished from the auxotrophic non-mucoid revertants arising from PAO568 during the course of the experiment. A preliminary experiment was performed to compare the growth rates of PAO547 and PAO548 in shaken and unshaken NB cultures incubated at $37^{\circ} \mathrm{C}$. Results obtained for shaken cultures of both strains indicated a generation time during the exponential growth phase of $25.0( \pm 2 \cdot 0) \mathrm{min}$. In unshaken culture, PAO547 and PAO548 showed similar growth curves with a mean generation time for both strains of 30.0 $( \pm 4 \cdot 0)$ min during the first $6 \mathrm{~h}$ growth. From these results it was concluded that PAO548 exhibited similar growth characteristics to PAO547 in NB culture.

Growth curves were then obtained for PA0568 and PAO548 grown competitively in unshaken and shaken cultures (Fig. 1). The viable counts of PAO568 were determined by counting the mucoid colonies on NA plates, whereas colonies of PAO548 were counted on MA.

In unshaken culture, during the period of maximum growth from 1.5 to $6 \mathrm{~h}$, the mean generation times of PAO568 and PAO548 were 37.5 and 30.5 min, respectively. Within the limits of probable error this allows for only a slight growth rate advantage for the nonmucoid strain during this period. After $6 \mathrm{~h}$, however, as the growth rate of the mucoid strain approached zero, there was clear evidence of a growth rate advantage for PAO548 as it continued to grow until $24 \mathrm{~h}$. During the period between 6 and $12 \mathrm{~h}$ the mean generation time of PAO568 was $97.5 \mathrm{~min}$ compared with $60.0 \mathrm{~min}$ for the non-mucoid strain. The percentage of colony-forming units of PAO548 in the unshaken culture increased from $0.2 \%$ at the beginning of the experiment to $6.0 \%$ after $12 \mathrm{~h}$ incubation and reached $52 \%$ after $24 \mathrm{~h}$.

In contrast, the growth rate advantage of PAO548 over the mucoid strain was less evident in shaken culture. The generation time of PAO568 between 1.5 and $6 \mathrm{~h}$ was $26.7 \mathrm{~min}$ compared with $23.7 \mathrm{~min}$ for PAO548. After $6 \mathrm{~h}$ the growth rate of PAO568 approached zero only slightly ahead of the non-mucoid strain. By $12 \mathrm{~h}$ the percentage of colony-forming 


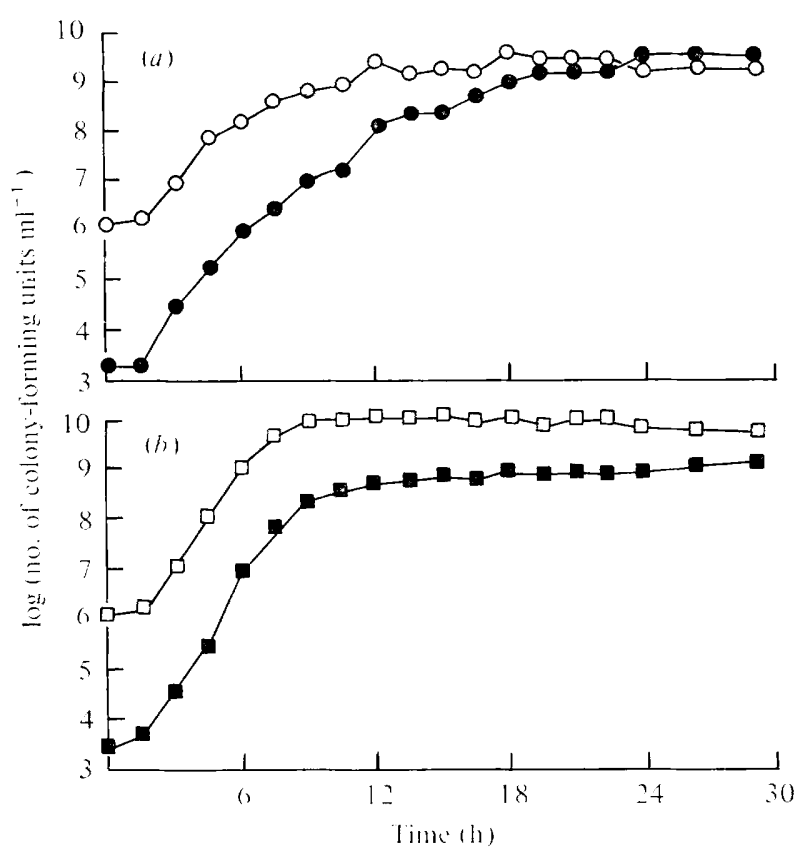

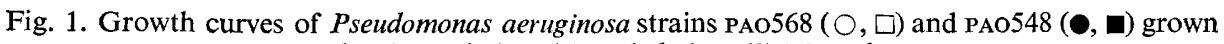
together in unshaken $(a)$ and shaken $(b)$ NB cultures.

units of PAO548 in the shaken culture was $3 \cdot 1 \%$ and by $24 \mathrm{~h}$ had only risen to $13 \cdot 4 \%$.

We have previously provided evidence that mucoid variants can arise from non-mucoid $P$. aeruginosa via spontaneous mutation and that mucoid variants can be isolated in vitro by selecting for increased resistance to carbenicillin (Govan, 1976a, b; Govan \& Fyfe, 1978). Conversely, we now suggest that the instability of mucoid $P$. aeruginosa also results from spontaneous mutation, back to the non-mucoid form which then has a selective growth advantage in unshaken cultures.

Mian et al. (1978) observed that during continuous culture of mucoid $P$. aeruginosa, non-mucoid variants arose in the mucoid population and increased as a proportion of the total number of organisms. The rate of this increase was thought to be consistent with mutation followed by selective advantage of the non-mucoid strain.

The effect of exopolysaccharide synthesis per se on the growth and instability of mucoid $P$. aeruginosa grown without shaking was suggested by further observations. Exopolysaccharide could be detected, by alcohol precipitation (Evans \& Linker, 1973), in culture supernates from shaken and unshaken NB cultures from the early stationary phase. In contrast, no exopolysaccharide could be detected when PAO568 was grown in minimal broth (Govan, 1976b) or anaerobically in NB supplemented with $0.4 \%(\mathrm{w} / \mathrm{v}) \mathrm{KNO}_{3}$, and under these conditions PAO568 remained stable.

Several explanations can be offered to explain the differences in the stability of mucoid organisms in shaken or unshaken culture. It is possible that physical dispersion of cellbound polysaccharide in shaken culture results in improved oxygen and nutrient uptake. Another possible explanation is that the polysaccharide surrounding the cell acts as a diffusion barrier to oxygen transfer and results in a higher growth rate for mucoid organisms in the increased dissolved oxygen concentration maintained in shaken culture.

This work was supported by a research grant from the Jeffrey Charitable Trust in association with the Cystic Fibrosis Research Trust. 


\section{REFERENCES}

Evans, L. R. \& Linker, A. (1973). Production and characterization of the slime polysaccharide of Pseudomonas aeruginosa. Journal of Bacteriology 116, 915-924.

Govan, J. R. W. (1975). Mucoid strains of Pseudomonas aeruginosa: the influence of culture medium on the stability of mucus production. Journal of Medical Microbiology 8, 513-522.

Govan, J. R. W. (1976a). Antibiotic therapy and cystic fibrosis: increased resistance of the mucoid Pseudomonas aeruginosa to carbenicillin. Journal of Antimicrobial Chemotherapy 2, 215-217.

Govan, J. R. W. $(1976 b)$. Genetic studies on mucoid Pseudomonas aeruginosa. Proceedings of the Society for General Microbiology 3, 187.

Govan, J. R. W. \& Fyfe, J. A. M. (1978). Mucoid Pseudomonas aeruginosa and cystic fibrosis: resistance of the mucoid form to carbenicillin, flucloxacillin and tobramycin and the isolation of mucoid variants in vitro. Journal of Antimicrobial Chemotherapy 4, 233-240.

Henriksen, S. D. (1948). Some unusual mucoid organisms. Acta pathologica et microbiologica scandinavica 25, 485-492.

Holloway, B. W. (1955). Genetic recombination in Pseudomonas aeruginosa. Journal of General Microbiology 13, 572-581.

Holloway, B. W., Monk, M., Hodgins, L. \& FARGIE, B. (1962). Effects of radiation on transduction in Pseudomonas aeruginosa. Virology 18, 89-94.
Krishnapillai, V. (1971). A novel transducing phage. Its role in recognition of a possible new host controlled modification system in Pseudomonas aeruginosa. Molecular and General Genetics 114, 134-143.

Luria, S. E. \& Delbruck, M. (1943). Mutations of bacteria from virus sensitivity to virus resistance. Genetics 28, 491-511.

Martin, D. R. (1973). Mucoid variation in Pseudomonas aeruginosa induced by the action of phage. Journal of Medical Microbiology 6, 111-118.

Mian, F. A., Jarman, T. R. \& Righelato, R. C. (1978). Biosynthesis of exopolysaccharide by Pseudomonas aeruginosa. Journal of Bacteriology 134, 418-422.

Sonnenschein, C. (1927). Die Mucosus-Form des Pyocyaneus-Bakteriums, Bacterium pyocyaneum mucosum. Zentralblatt für Bakteriologie, Parasitenkunde und Infektionskrankheiten (Abteilung I, Orig.) 104, 365-373.

Stanisich, V. \& Holloway, B. W. (1969). Conjugation in Pseudomonas aeruginosa. Genetics 61, 327-339.

Vogel, H. J. \& BonNer, D. M. (1956). Acetylornithinase of Escherichia coli: partial purification and some properties. Journal of Biological Chemistry 218, 97-106.

ZiERDT, C. H. \& SCHMidT, P. J. (1964). Dissociation in Pseudomonas aeruginosa. Journal of Bacteriology 87, 1003-1010. 